\title{
Information Retrieval and Machine Learning for Probabilistic Schema Matching.
}

\author{
Henrik Nottelmann \\ University of Duisburg-Essen \\ Lotharstrasse 65 \\ 47048 Duisburg, Germany \\ nottelmann@uni-duisburg.de
}

\author{
Umberto Straccia \\ ISTI-CNR \\ Via G. Moruzzi 1 \\ 56124 Pisa, Italy \\ straccia@isti.cnr.it
}

\begin{abstract}
Schema matching is the problem of finding correspondences (mapping rules, e.g. logical formulae) between heterogeneous schemas. This paper presents a probabilistic framework, called sPLMap, for automatically learning schema mapping rules. Similar to LSD, different techniques, mostly from the IR field, are combined. Our approach, however, is also able to give a probabilistic interpretation of the prediction weights of the candidates, and to select the rule set with highest matching probability.
\end{abstract}

Categories and Subject Descriptors: H.2.1 [Database Management]: Logical Design - Schema and subschema

General Terms: Theory, Experimentation

Keywords: sPLMap, schema matching, predicate logics, probability theory

\section{INTRODUCTION}

Combining heterogeneous data from different sources, i.e. transforming data structured under one schema into data structured under a different schema, is an old but emerging problem in the "information age" (e.g. for federations of existing digital libraries like the ACM DL or CiteSeer). This problem is currently under investigation in the context of information integration [3] and data exchange [2]. When schemas change frequently over time, or a large amount of schemas is involved, automatic mapping generation (called schema matching) is mandatory.

This paper describes the sPLMap framework (probabilistic, logicbased mapping between schemas) [4, 5] for automatic schema matching, which is founded on information retrieval and machine learning techniques. From well-known approaches like LSD [1], it borrows the idea of combining several specialized components (called "classifiers") for finding the best mapping, e.g. based on attribute name comparison or comparing properties of the underlying

*This work is supported in part by the DFG (grant BIB47 DOuv 02-01, project "Pepper") and in part by ISTI-CNR (project "Distributed Search in the Semantic Web").

Copyright is held by the author/owner.

CIKM'05, October 31-November 5, 2005, Bremen, Germany.

ACM 1-59593-140-6/05/0010. data instances. The major improvements over LSD are the support for data types (e.g. text, names, different date formats) in the matching process, and the usage of probability theory for estimating the degree of correctness of a learned rule. Thus, it provides a sound theoretical justification for its (optimum) selection, and a measure for the quality of a learned schema mapping.

In the following, a schema $\mathbf{R}=\left\langle R_{1}, \ldots, R_{n}\right\rangle$ is a tuple of binary relations $R_{i}$ containing document identifiers and attribute values (belonging to a data type, e.g. "Text", "Name", "Year" or "DateISO8601"). This structure corresponds to a linear list of multivalued schema attributes. The instance of an attribute $R_{i}$ is also denoted by $R_{i}$, and, similarly, $\mathbf{R}$ is used for the schema and the corresponding schema instance.

A schema mapping rule has the form $S_{i} \rightarrow T_{j}$. Formally, it specifies that pairs of document identifier and attribute values have to be copied from source instance $S_{i}$ into target instance $T_{j}$. Let $\Sigma_{j}$ be a set of rules with common rule head $T_{j}$. Then, $\widehat{T}_{j}=\bigcup_{i_{k}} S_{i_{k}}$ denotes the result of applying all rules from $\Sigma_{j}$ onto the source instance $\mathbf{S}$; thus, $\widehat{T}_{j}$ is an instance over the target attribute $T_{j} . \widehat{\mathbf{T}}=\left\langle\widehat{T}_{1}, \ldots, \widehat{T}_{t}\right\rangle$ denotes the instance derived by applying $\Sigma$ onto $\mathbf{S}$. Typically, the set $\Sigma$ of mapping rules on which $\widehat{T}_{j}$ and $\widehat{\mathbf{T}}$ depend is clear from the context and left out.

\section{LEARNING SCHEMA MAPPINGS}

Consider a target schema $\mathbf{T}=\left\langle T_{1}, \ldots, T_{t}\right\rangle$ and a source schema $\mathbf{S}=\left\langle S_{1}, \ldots, S_{s}\right\rangle$ together with two instances (which do not necessarily describe the same documents). The goal is to find the "best" set of mapping rules $\Sigma$ which maximizes the probability $\operatorname{Pr}(\Sigma, \mathbf{T}, \mathbf{S})$ that the tuples in $\mathbf{T}$ and in the mapping result $\widehat{\mathbf{T}}$ are 'similar', that is, given a random tuple in $\mathbf{T}$, estimate the probability that it is a tuple in $\widehat{\mathbf{T}}$ and vice-versa.

Each $\Sigma$ can be partitioned into $t$ sets $\Sigma_{1}, \ldots, \Sigma_{t}$ (where any $\Sigma_{j}$ contains only rules with rule head $T_{j}$ ). As the rules in these sets operate independently onto different target attributes, we obtain:

$$
\operatorname{Pr}(\Sigma, \mathbf{T}, \mathbf{S})=\prod_{j=1}^{t} \operatorname{Pr}\left(\Sigma_{j}, \mathbf{T}, \mathbf{S}\right) .
$$

Now, $\operatorname{Pr}\left(\Sigma_{j}, \mathbf{T}, \mathbf{S}\right)$ is estimated as the probability that a tuple in $\widehat{T}_{j}$ is also in $T_{j}$, and vice-versa:

$$
\operatorname{Pr}\left(\Sigma_{j}, \mathbf{T}, \mathbf{S}\right)=\operatorname{Pr}\left(T_{j} \mid \widehat{T}_{j}\right) \cdot \operatorname{Pr}\left(\widehat{T}_{j} \mid T_{j}\right)=\operatorname{Pr}\left(\widehat{T}_{j} \mid T_{j}\right)^{2} \cdot \frac{\left|T_{j}\right|}{\left|\widehat{T}_{j}\right|} .
$$

Unfortunately, there are exponentially many possible sets $\Sigma_{j}$. For computational simplification, we assume that $S_{i_{1}}$ and $S_{i_{2}}$ are dis- 


\begin{tabular}{llll}
\hline \multicolumn{2}{l}{ Schema-based } & \multicolumn{2}{c}{ Content-based } \\
\hline$C L_{N}$ & same name & $C L_{L}$ & value overlap \\
$C L_{S}$ & same name stem & $C L_{k N N}$ & kNN \\
$C L_{D}$ & same data type & $C L_{B}$ & Naive Bayes \\
& & $C L_{K L}$ & KL-distance \\
\hline
\end{tabular}

Table 1: Classifiers

joint for $i_{1} \neq i_{2}$. This leads us to the approximation:

$$
\operatorname{Pr}\left(\widehat{T}_{j} \mid T_{j}\right) \approx \sum \operatorname{Pr}\left(S_{i_{k}} \mid T_{j}\right) .
$$

Thus, in order to compute $\operatorname{Pr}\left(\Sigma_{j}, \mathbf{T}, \mathbf{S}\right)$, the main task is to compute the $O(s \cdot t)$ probabilities $\operatorname{Pr}\left(S_{i} \mid T_{j}\right)$. Similar to LSD [1], we combine different classifiers $C L_{1}, \ldots, C L_{n}$ (see table 1 for classifiers we use). Each classifier outputs a conditional probability $\operatorname{Pr}\left(S_{i} \mid T_{j}, C L_{k}\right)$ as an approximation of $\operatorname{Pr}\left(S_{i} \mid T_{j}\right)$. The final probability $\operatorname{Pr}\left(S_{i} \mid T_{j}\right)$ is computed by combining the classifier predictions $\operatorname{Pr}\left(S_{i} \mid T_{j}, C L_{k}\right)$ in a weighted sum:

$$
\operatorname{Pr}\left(S_{i} \mid T_{j}\right) \approx \sum_{k=1}^{n} \operatorname{Pr}\left(S_{i} \mid T_{j}, C L_{k}\right) \cdot \operatorname{Pr}\left(C L_{k}\right) .
$$

In a first step, each classifier $C L_{k}$ computes an initial weight $w\left(S_{i}, T_{j}, C L_{k}\right)$, which is then normalized (due to heterogeneous scales) into the probability $\operatorname{Pr}\left(S_{i} \mid T_{j}, C L_{k}\right)=f\left(w\left(S_{i}, T_{j}, C L_{k}\right)\right)$. In a first normalization step, an arbitrary function can be used, e.g. linear or logistic functions. A second normalization ensures that the final values for $\operatorname{Pr}\left(S_{i} \mid T_{j}, C L_{k}\right)$ and for $\operatorname{Pr}\left(T_{j} \mid S_{i}, C L_{k}\right)$ are in $[0,1]$.

Finally, the probability $\operatorname{Pr}\left(C L_{k}\right)$ describes the probability that we rely on the judgment of classifier $C L_{k}$. We simply set $\operatorname{Pr}\left(C L_{k}\right)=\frac{1}{n}$ (i.e., we average over all classifiers). Alternatively, one could aim at learning these probabilities for each classifiers individually.

\section{EVALUATION}

This section briefly describes some experimental results; more results can be found in $[4,5]$. We use a 2.382 document BibTeX collection (BIBDB) with a large overlap in attribute names, and an Open Archive collection of the Library of Congress (LOC) with 1.337 entries, available in MARC 21 and Dublin Core. Both collections are split randomly into three sub-collections of approximately the same size (two for learning, one for evaluation). Classifiers from table 1 are used in isolation as well as in combination. As all LOC attributes use the same data type "Text", and do not share common names, only the content-oriented classifiers are used for this collection.

The results in table 2 use the typical IR measures precision, recall and F-measure. For BIBDB, the combination of all classifiers yields $82 \%$ of precision and recall. According to the F-measure, the best schema classifier are $C L_{N}$ and $C L_{S}$ and the worst is $C L_{D}$, while the best content classifier is $C L_{L}$ and the worst is $C L_{K L}$. For LOC, the combination of all classifiers yields $64 \%$ of precision and $22 \%$ of recall only. The best content classifier is $C L_{k N N}$ (which slightly outperforms the combination) and the worst is $C L_{K L}$.

There is no general best classifier. Which classifier performs well always depends on the concrete schemas (and their instances). The experiments show for BIBDB (and other similar collections we cannot report here due to space limitations) satisfactory results for the combination of all classifiers, while the performance is worse for the difficult LOC collection (where the DC attributes often contain text which equals the concatenation of strings from multiple MARC 21 attributes). Typically, the name-based classifiers provide good precision and rather low recall, while using data types (a) BIBDB

\begin{tabular}{llll}
\hline & Prec & Rec & $F$ \\
\hline$C L_{N}$ & 1.0000 & 0.6364 & 0.7778 \\
$C L_{S}$ & 1.0000 & 0.6364 & 0.7778 \\
$C L_{D}$ & 0.2632 & 0.9091 & 0.4082 \\
$C L_{k N N}$ & 0.5455 & 0.5455 & 0.5455 \\
$C L_{B}$ & 0.5000 & 0.4545 & 0.4762 \\
$C L_{L}$ & 0.8750 & 0.6364 & 0.6368 \\
$C L_{K L}$ & 0.2353 & 0.7273 & 0.3556 \\
all schema & 0.4348 & 0.9091 & 0.5882 \\
all content & 0.4211 & 0.7273 & 0.5333 \\
all & 0.8182 & 0.8182 & 0.8182 \\
\hline
\end{tabular}

(b) LOC

\begin{tabular}{llll}
\hline & Prec & Rec & $F$ \\
\hline$C L_{k N N}$ & 0.6667 & 0.2439 & 0.3571 \\
$C L_{B}$ & 0.4375 & 0.1707 & 0.2456 \\
$C L_{L}$ & 0.8000 & 0.1951 & 0.3137 \\
$C L_{K L}$ & 0.1714 & 0.2927 & 0.2162 \\
all content & 0.6429 & 0.2195 & 0.3273 \\
\hline
\end{tabular}

Table 2: Effectiveness evaluation

perform worse. Content-oriented classifiers proved to be very useful for schema matching (w.r.t. both precision and recall), which is important in general settings where schema names alone are not sufficient to find matching attributes. Here, $\mathrm{kNN}$ and Naive Bayes provide high precision. $C L_{K L}$ yields low precision but can improve recall when combined with other classifiers. The experiments further showed that combining classifiers can clearly increase the matching quality.

\section{CONCLUSION AND OUTLOOK}

We have presented sPLMap, a Probabilistic, Logic-based formal framework for the important schema matching problem. The peculiarity of our approach is that it combines neatly machine learning, information retrieval and heuristic techniques for learning a set of mapping rules. The framework has already been extended towards uncertain rules $[4,5]$. In future, we plan to develop and evaluate additional classifiers $C L_{K}$, and learn the probabilities $\operatorname{Pr}\left(C L_{k}\right)$. This framework can easily be extended towards more complex rules, which e.g. combine the content of several attributes, and to new application areas like ontologies.

\section{REFERENCES}

[1] A. Doan, P. Domingos, and A. Y. Halevy. Reconciling schemas of disparate data sources: a machine-learning approach. In ACM SIGMOD international conference on Management of data, 2001.

[2] R. Fagin, P. G. Kolaitis, R. Miller, and L. Popa. Data exchange: Semantics and query answering. In International Conference on Database Theory (ICDT), 2003.

[3] M. Lenzerini. Data integration: a theoretical perspective. In ACM SIGMOD-SIGACT-SIGART symposium on Principles of database systems (PODS), 2002.

[4] H. Nottelmann and U. Straccia. A probabilistic approach to schema matching. Technical Report 2004-TR-60, Istituto di Scienza e Tecnologie dell'Informazione, Consiglio Nazionale delle Ricerche, Pisa, Italy, 2004.

[5] H. Nottelmann and U. Straccia. sPLMap: A probabilistic approach to schema matching. In European Conference on Information Retrieval Research (ECIR), 2005. 